\title{
TRIGGERING THE BOILING CRISIS: A STUDY OF THE DRY SPOT SPREADING MECHANISM
}

\author{
Vladislav Janeček ${ }^{1} \mathcal{E}$ Vadim S. Nikolayev ${ }^{2, *}$
}

${ }^{1}$ Physique et Mécanique des Milieux Hétérogènes, UMR 7636 ESPCI - CNRS - Univ. ParisDiderot - Univ. P.M. Curie, 10 rue Vauquelin, 75005 Paris, France; Present address: ArcelorMittal Global RED, Voie Romaine, BP 30320, Maizières-lès-Metz, 57283, France

${ }^{2}$ Service de Physique de l'Etat Condensé, CNRS UMR 3680, IRAMIS/DSM/CEA Saclay, 91191 Gif-sur-Yvette, France

*Address all correspondence to Vadim S. Nikolayev, E-mail: vadim.nikolayev@cea.fr

In this article we describe the boiling crisis (departure from nucleate boiling) as a transition between two regimes of bubble growth: "bubble departure" and "bubble spreading," which occur for small and large heat fluxes, respectively. The threshold heat flux is the critical heat flux (CHF). Our assumptions are based on existing experimental observations. The boiling crisis is assumed to be triggered by spreading of dry spots under individual bubbles that occurs due to the apparent contact angle growth, when the apparent contact angle attains $90^{\circ}$. Such a mechanism is studied via numerical simulation of the single bubble growth. The apparent contact angle is obtained from the hydrodynamic modeling of the vicinity of the triple contact line (microregion). The microregion model is based on the lubrication approximation extended to treat high interface slopes. The microregion model is compared to previous experimental and numerical data obtained for moderate heater superheating values. The macroregion is modeled within a simplified 2D quasistationary approach that neglects the fluid motion. The CHF obtained from such a formulation is calculated as a function of gravity level and the heater wetting properties. The results agree qualitatively with existing experimental data.

KEY WORDS: CHF, DNB, boiling crisis, bubble growth, bubble departure, contact line

\section{INTRODUCTION}

When, during boiling, the supplied heat flux exceeds a critical value (the critical heat flux, CHF) the vapor bubbles on the heating surface abruptly form a film that thermally insulates the heater from the liquid. The heat transfer is blocked and the temperature of the heater rapidly grows. This transition is known under the names of "boiling crisis" (BC) and "departure from nucleate boiling."

In the engineering literature, the term "critical heat flux" (CHF) is often used both as a synonym to the term "boiling crisis" (BC) and as a particular heat flux value. To avoid the ambiguity, we use the term CHF only to designate the threshold heat flux.

Correct CHF estimation requires a clear understanding of the physical phenomena that trigger it. Among several dozens of existing models of BC [see Buyevich (1999); Dhir (1998); Theofanous et al. (2002) for their discussion and classification], the Zuber model is the only one that can be considered as a physical theory, the others being based on multiple assumptions which are merely empirically based without a solid scientific justification. According to Zuber's model, vapor columns form at nucleation sites on the heater. The vapor moves upward while the liquid moves to the bottom of column where the evaporation occurs. This counter-flow motion induces the Kelvin-Helmholtz instability, which is supposed to cause destabilization of the whole system and formation of a vapor film on the heater. This transition occurs when the vapor velocity exceeds a threshold resulting in the following famous expression:

$$
q_{C H F} \sim H\left[\sigma g\left(\rho_{L}-\rho_{V}\right) \rho_{V}^{2}\right]^{1 / 4},
$$




\section{NOMENCLATURE}

BC boiling crisis

BEM boundary element method

Bo $\quad$ Bond number $=g\left(\rho_{L}-\rho_{V}\right) R_{0}^{2} / \sigma$

CHF critical heat flux (also as a subscript)

CL contact line

Fo $\quad$ Fourier number $=\alpha \Delta t / R_{0}^{2}$

Ja Jakob number $=q_{0} R_{0} /\left(\alpha_{L} H \rho_{V}\right)$

$\vec{e} \quad$ unit vector directed along an axis

$\vec{n} \quad$ internal to the bubble unit normal vector to its surface

$c_{p} \quad$ liquid specific heat, $[\mathrm{J} /(\mathrm{kg} \mathrm{K})]$

$G \quad$ correction coefficient, see Appendix

$g \quad$ gravity acceleration, $\left[\mathrm{m} / \mathrm{s}^{2}\right]$

$H \quad$ latent heat, $[\mathrm{J} / \mathrm{kg}]$

$h$ height of the interface in the

microregion, [m]

$J \quad$ mass evaporation flux, $\left[\mathrm{kg} /\left(\mathrm{m}^{2} \mathrm{~s}\right)\right]$

$j \quad$ volume heat supply, $\left[\mathrm{W} / \mathrm{m}^{3}\right]$

$K \quad$ curvature, $\left[\mathrm{m}^{-1}\right]$

$k$ heat conductivity, $[\mathrm{W} /(\mathrm{m} \cdot \mathrm{K})]$

$L \quad$ bubble contour half length, [m]

$l_{s} \quad$ hydrodynamic slip length, [m]

$Q \quad$ integrated heat flux, [W/m]

$q \quad$ heat flux, $\left[\mathrm{W} / \mathrm{m}^{2}\right]$

$R$ bubble radius, [m]

$r \quad$ local wedge coordinate (Fig. 2), [m]

$R^{i} \quad$ interfacial thermal resistance, [( $\left.\left(\mathrm{K} \mathrm{m}^{2}\right) / \mathrm{W}\right]$

$s \quad$ curvilinear coordinate, $[\mathrm{m}]$

$T$ temperature, [K]

$t$ time, [s]

$U$ volume flux, $\left[\mathrm{m}^{3} / \mathrm{s}\right]$

$u \quad$ local slope of the liquid-gas interface $=d h / d x$

$V \quad$ bubble $2 \mathrm{D}$ half volume, $\left[\mathrm{m}^{2}\right]$

$x, y$ coordinates, [m]

\section{Greek Symbols}

$\alpha \quad$ thermal diffusivity, $\left[\mathrm{m}^{2} / \mathrm{s}\right]$

$\Delta p \quad$ vapor-liquid interface pressure jump

$=p_{V}-p_{L},[\mathrm{~Pa}]$

$\Delta T \quad$ microregion wall superheating, [K]

$\Delta t \quad$ time step, [s]

$\delta \quad$ arc length, [m]

$\gamma \quad$ Marangoni coefficient, [N/(m K)]

$\mu \quad$ liquid shear viscosity, [Pa s]

$\Omega \quad$ boundary separating domains

$\phi \quad$ local slope angle of the liquid-gas interface

$\psi \quad$ reduced temperature

$\rho \quad$ density, $\left[\mathrm{kg} / \mathrm{m}^{3}\right]$

$\sigma \quad$ surface tension, $[\mathrm{N} / \mathrm{m}]$

$\theta \quad$ contact angle

$\xi \quad$ reduced curvilinear coordinate

$\zeta \quad$ reduced heat flux

$\begin{array}{ll}\text { Subscripts and Superscripts } \\ \text { app } & \text { apparent } \\ d & \text { dry } \\ d e p & \text { at bubble departure } \\ i & \text { vapor-liquid interface } \\ \text { inf } & \text { at } x \rightarrow \infty \\ L & \text { liquid } \\ M & \text { of point } \mathrm{M}, \text { cf. Fig. } 2 \\ \text { macro } & \text { macroregion } \\ \text { micro } & \text { microregion } \\ r & \text { recoil } \\ S & \text { solid heater } \\ \text { sat } & \text { saturation } \\ V & \text { vapor } \\ w & \text { wetted } \\ x, y & \text { components of a vector } \\ 0 & \text { at } t=0 \text { or } y=0\end{array}$

where $H$ is the latent heat, $\rho_{L}\left(\rho_{V}\right)$ is the density of the liquid (gas) phase, $\sigma$ is the surface tension, and $g$ is the gravity acceleration. Equation (1) was first obtained by Kutateladze (1950) out of dimensional analysis. While this expression seems more or less compatible with the experimental data sets, Zuber's model itself is questionable. Indeed, the vapor column morphology of boiling is quite rarely observed while the BC occurs inevitably for all morphologies of boiling, for pool as well as for flow boiling. Besides, many experimental results, in particular those obtained in low gravity (Kim et al., 2002), cannot be fitted by Eq. (1). Other physical phenomena should then be responsible for the triggering of BC. A strong dependence of CHF on the wetting properties and the surface state of the heater (Dhir, 
1998) suggests a phenomenon at the level of the triple (vapor-liquid-solid) contact line rather than a phenomenon related to the bulk hydrodynamics. Until the mid 1990s, it was considered that a thin liquid film (macrolayer) covers continuously the heater, thus separating it from the bubbles. Recent advance in the optical and infrared observation techniques of $\mathrm{BC}$ through the transparent heater with rapid cameras evidenced an important role of growth of the dry heater area; see, e.g., Gong et al. (2014); Kandlikar (2001); Kannengieser et al. (2014); Theofanous et al. (2002). More and more researchers in this field consider the dry area growth to be the key phenomenon; see, e.g., Gong et al. (2014); Kannengieser et al. (2014); Nikolayev and Beysens (1999); Yagov (2014).

It is important to understand how the drying impacts the $\mathrm{BC}$ triggering. Either it is triggered by the growth of dry spots under individual vapor bubbles (Nikolayev and Beysens, 1999; Nikolayev et al., 2001) that coalesce later or BC occurs as a result of a collective phenomenon of the avalanche-like multiple bubble coalescence (Lloveras et al., 2012). This is still an open question. A thorough analysis (Bricard et al., 1997) of this second scenario has shown a necessity of the repetitive (at least 30-40 times) formation of aggregates of 2-3 coalesced bubbles at the same neighboring nucleation spots. This is not a likely event because in order to coalesce, (i) the bubbles need to grow close to each other, which is prohibited by their thermal interaction (Kenning and Del Valle, 1981; Pasamehmetoglu and Nelson, 1991), and (ii) the strong lubrication forces between their interfaces need to be overcome (Yiantsios and Davis, 1991), which means that either the bubbles are densely crowded or strongly pinned at their nucleation sites. More recent experimental studies (Chatpun et al., 2004; Golobic et al., 2011) corroborate these considerations. In the present paper we develop the first scenario that has been suggested theoretically (Nikolayev and Beysens, 1999) and confirmed by observation of growth of individual dry spots through the transparent heater (Chung and No, 2003; Kandlikar and Steinke, 2002; Kannengieser et al., 2014) or via infrared visualization (Jung et al., 2014). It is however very difficult to observe experimentally the dynamics of BC (Theofanous et al., 2002) because of fast motion of the liquid-gas interfaces and temperature gradients that cause strong optical aberrations (Cooper and Chandratilleke, 1981). To our knowledge, the dynamics of BC that clearly showed its progression has been observed only at nearly critical pressure where the weak heat diffusion slows down the boiling process and where the CHF value is small (Nikolayev et al., 2006a,b) so that hindering the optical observations violent fluid motion is absent. Such experiments had, however, to be performed in the weightlessness to avoid the gravity flattening of all gas-liquid interfaces. The gravity influence (appearing mainly because of the surface tension decrease) is progressively stronger as the critical pressure is approached. In these experiments, it has been shown that the spreading of dry spots under individual bubbles precedes their coalescence and gives rise to the fast heater temperature increase. The "vapor spreading" scenario has thus been evidenced.

The main cause of the dry spot growth is the increase of the apparent contact angle; see, e.g., Ajaev (2005); Moosman and Homsy (1980); Nikolayev (2010); Raj et al. (2012); Stephan and Hammer (1994); Wayner et al. (1976). For bubbles in boiling, the relation between the apparent contact angle and the size of the dry spot was evidenced both experimentally (Chung and No, 2003; Kandlikar and Steinke, 2002) and by numerical simulation (Kunkelmann and Stephan, 2009; Mukherjee and Dhir, 2004; Nikolayev et al., 2001; Son et al., 1999). It has been shown (Garrabos et al., 2001; Nikolayev et al., 2006b) that at nearly critical pressure where the hydrodynamic flow is very slow, the vapor recoil is the main cause of the apparent contact angle increase because of its relative strength with respect to the surface tension. Far from the critical pressure, their balance (Nikolayev et al., 2006b) leads to an expression similar to Eq. (1).

A somewhat different experimental approach (Gong et al., 2011) aimed to study the dynamics of formation and spreading of a single dry spot in a liquid film. The film geometry is more relevant in the flow boiling where BC occurs via the dryout of such a film (called sometimes macrolayer) that forms between the heater and the moving vapor slugs. This scenario is actually similar to that considered here because the dry spot growth occurs due to the same microscopic reason: the increase of the apparent contact angle. In this work we address the case of the pool boiling where the bubble geometry is relevant.

An attempt has already been made (Nikolayev et al., 2001) to simulate numerically the spreading of a dry spot under a vapor bubble. However, in the evaluation of the apparent contact angle, the effect of the viscous pressure drop caused by the fluid flow near the triple contact line was neglected with respect to the vapor recoil effect. The action of these two effects is similar. However our a posteriori estimations (Janeček, 2012) have shown that for the pressures (at least 20\%) lower than the critical pressure, the viscous pressure drop cannot be ignored because its effect may be 
comparable and even larger than that of the vapor recoil at low pressures. Recently, a contact line model accounting for these both effects has been developed by us (Janeček and Nikolayev, 2012, 2013). One of the purposes of the present study is to extend our contact line model for high apparent contact angles. Then its coupling with a simple quasistatic bubble growth model is performed. Unlike the existing studies where the microregion was implemented as a subgrid model, a rigorous coupling between the micro and macro regions is proposed here. We also present a parametric analysis of the CHF obtained in the framework of such a simulation applied to the proposed BC scenario.

\section{PROBLEM STATEMENT}

The dry spot formed on the heater under the vapor bubble depends on its shape defined by its apparent contact angle $\theta_{a p p}$. Under $\theta_{a p p}$ we mean the contact angle defined on a scale comparable to the bubble size (Fig. 1). This scale is called hereafter "macroscopic" to distinguish it from the microscopic scale of the contact line (CL) phenomena. The apparent contact angle may differ from its equilibrium (usually small) value $\theta_{\text {micro }}$ as will be shown below. It depends on the local heater temperature at the CL position that varies with time. Therefore, $\theta_{\text {app }}$ changes (usually, increases) during the bubble growth.

\subsection{Boiling Crisis Scenario}

Based on the experimental observations discussed above, the CHF can be calculated as follows. At low heat fluxes $\theta_{\text {app }}$ remains low throughout the bubble growth on the heater; the bubble grows and departs. At some heat flux, the apparent contact angle may attain $90^{\circ}$ during the bubble growth. Such an event is very important for several reasons. First, the dry area attains its maximum value with respect to the bubble size, which leads to the heater temperature rise. Second, the bubble adhesion to the heater increases, which facilitates the bubble coalescence. Third, and the most important, the dry areas under bubbles coalesce simultaneously with the bubble volume coalescence, which leads to the fast heater temperature rise.

In the opposite case $\theta_{a p p}<90^{\circ}$, the coalescence of bubble volumes does not result in immediate dry spot coalescence and growth; the heater continues to be cooled under the newly formed bubble (because of the latent heat consumption) even after the coalescence of bubble volumes. The dry spot coalescence is slow because large viscous

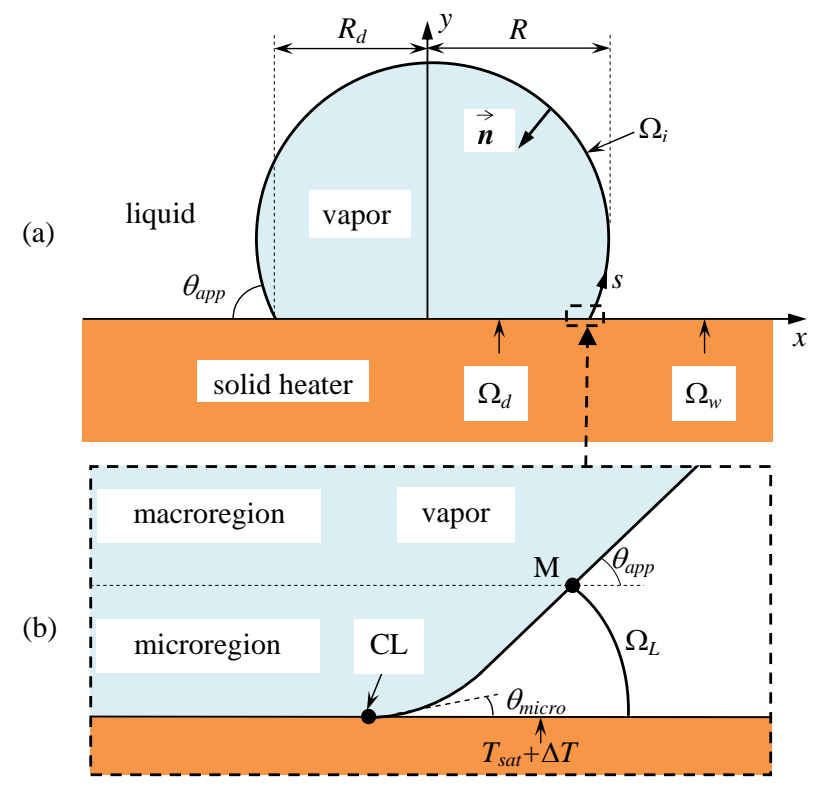

FIG. 1: Vapor bubble on the heater surrounded by liquid. The macroregion (a) and microregion (b) are shown. 
dissipation in the thin liquid film appearing in the CL vicinity and pinning at solid surface defects causes the slowness of the CL motion.

All these reasons suggest that the triggering of $\mathrm{BC}$ occurs when $\theta_{\text {app }}$ becomes larger than $90^{\circ}$ while the bubble grows on the heater. Therefore we postulate that CHF corresponds to the situation where $\theta_{\text {app }}$ attains $90^{\circ}$ exactly at the moment of bubble departure. Such an assumption is confirmed by the direct optical observations of Kannengieser et al. (2014).

Note that, within the quasistatic bubble shape considered below, the above criterion is equivalent to the equality of the dry spot radius and the bubble curvature radius at bubble departure. This latter criterion was adopted by Yagov (2014).

To prove an occurrence of such a phenomenon, a numerical simulation of growth of a single bubble is performed. The difficulty of such a task is its multiscale nature: the scales ranging from $\mathrm{mm}$ (bubble size) to $\mathrm{nm}$ (microscopic scale of the CL phenomena) need to be solved. For this reason, the computational domain is decomposed into two parts (cf. Fig. 1): (i) a region of vapor and liquid in the vicinity of CL called microregion and (ii) the rest of the simulation domain including both liquid and solid parts, called macroregion. The problems in these two regions are solved separately and coupled with an iterative procedure. By using the above assumption, the CHF will be determined and its dependence on some system parameters will be studied.

\subsection{Macro Region}

We consider the growth of a vapor bubble with free interface attached to the semi-infinite $(y<0)$ solid heater in the semi-infinite $(y>0)$ liquid domain in 2D; see Fig. 1 . Since the bubble is axially symmetrical, only a half of the whole domain at $x>0$ may be considered.

\subsubsection{Thermal Part of the Problem}

The heat transfer due to convection is neglected in this model problem; only the heat conduction in the liquid is accounted for. This corresponds to the slow bubble growth at high pressures and allows us using the boundary element method (BEM), which simplifies this problem a priori complicated because of the free bubble interface. Although such a model is quite rough (its deficiencies will be evident from the results), it is useful to illustrate how the coupling with the microregion model is performed to obtain the CHF from a numerical simulation. The vapor is assumed to be thermal insulator. Because of the assumed absence of hydrodynamic motion in the macroregion, the pressure is uniform both in the vapor and in the liquid. In the macroregion, the bubble interface is thus isothermal at $T_{\text {sat }}$.

A spatially homogeneous inside the solid heater volume heating (e.g., by the electric current that passes through the metal heater) is considered. The transient heat conduction equations for the solid and liquid domains,

$$
\begin{aligned}
& \frac{\partial T_{S}}{\partial t}=\alpha_{S} \nabla^{2} T_{S}+\frac{\alpha_{S}}{k_{S}} j(t), \quad y<0, \\
& \frac{\partial T_{L}}{\partial t}=\alpha_{L} \nabla^{2} T_{L}, \quad y>0
\end{aligned}
$$

will be solved. The boundary and initial conditions are defined similarly to Nikolayev et al. (2001). The case of saturated boiling is considered, which means that the temperature in the liquid far from the heater is $T_{\text {sat }}$, the saturation temperature for the given system pressure. The homogeneous initial temperature $T_{s a t}$ is assumed both in the solid and in the liquid. The temperature and heat flux continuity boundary conditions are imposed at the macroregion part of the solid-liquid interface, $\Omega_{L}$ and the solid-liquid portion of the boundary with the microregion (cf. Fig. 1).

Following Nikolayev et al. (2001), the volume heat supply in the solid phase is chosen in the form $j(t) \sim t^{-1 / 2}$, which results in the time constant heat flux far from the bubble $q(x \rightarrow \infty, y=0)$, denoted $q_{0}$ hereafter. This particular choice will allow us avoiding the influence of the varying in time heat flux on the bubble growth; $q_{0}$ can thus be used as a control parameter. It can be shown (Nikolayev et al., 2001) that

$$
T_{S}(x \rightarrow \infty, y=0)=T_{s a t}+\frac{2 q_{0}}{k_{L}} \sqrt{\frac{\alpha_{L} t}{\pi}} .
$$

Volume 2, Number 4, 2014 
Note that the heat flux to the liquid varies along the heater at a scale that corresponds to the heat diffusion length.

\subsubsection{Vapor Bubble Evolution Description}

The simulation starts with a small spherical bubble with the contact angle $\theta_{\text {micro }}$ and $R=R_{0}$. The bubble growth is controlled by the supplied to it heat,

$$
\begin{gathered}
H \rho_{L} \frac{d V}{d t}=Q_{\text {macro }}+Q_{\text {micro }} ; \\
Q_{\text {macro }}=\int_{\Omega_{i}} q_{L}^{i} d \Omega, \text { where } q_{L}^{i}=-\left.k_{L} \vec{n} \cdot \nabla T_{L}\right|_{\Omega_{i}}
\end{gathered}
$$

is the local heat flux from the liquid defined at the macroregion part $\Omega_{i}$ of the gas-liquid interface.

The bubble interface is free and is defined at each time moment by the normal interface stress balance

$$
K \sigma=\left(\rho_{L}-\rho_{V}\right) g y+\Delta p_{\text {macro }},
$$

where the first term on the right-hand side corresponds to the hydrostatic pressure. The left-hand side is the capillary pressure with $K$, the local curvature that varies along the interface. In the macroregion, both the liquid flow and the vapor recoil effects are neglected, which corresponds to the quasistatic approximation; the value of $\Delta p_{\text {macro }}$ is thus constant. This value is obtained by using the bubble volume value $V$ given by Eq. (5) as described below. To solve Eq. (6), $\theta_{a p p}$ needs to be prescribed. This value comes from the microregion calculation.

The bubble departure takes place when the lift-off forces acting on the bubble overcome its adhesion to the heater. The departure occurs when the contact pressure force (i.e., the reaction force of the solid, $2 R_{d} \Delta p_{\text {macro }}$ in the $2 \mathrm{D}$ case) becomes zero, which implies the condition (Nikolayev and Janeček, 2012)

$$
\left(\rho_{L}-\rho_{V}\right) V^{d e p} g=\sigma \sin \theta_{a p p}
$$

valid in 2D. In the most interesting case for us $\theta_{a p p} \leq 90^{\circ}$, the bubble departure occurs when $\Delta p_{\text {macro }}=0$ (in some cases, the departure may occur also when $R_{d}=0$ ) so that the conditions $\Delta p_{\text {macro }} \geq 0, R_{d}>0$ hold throughout the bubble growth. This means that a concave bubble profile (that corresponds to $\left.K\right|_{y=0}=\Delta p_{\text {macro }} / \sigma<0$ ) is impossible and $R_{d}^{d e p}>0$ similarly to many other bubble growth simulations. Because of the bubble convexity, the condition $\theta_{\text {app }}$ $=90^{\circ}$ is equivalent to the condition $R_{d}=R$. Note that in reality, just before the departure, $R_{d}$ may rapidly shrink to zero or the bubble may exhibit transiently concave "bottleneck" profiles. However, such hydrodynamically controlled phenomena cannot be described within the quasistatic macroregion model.

\subsection{Microregion}

Conventional microregion modeling (Ajaev, 2005; Moosman and Homsy, 1980; Stephan and Hammer, 1994; Wayner et al., 1976) addresses the complete wetting regime, applicable only to low-energy fluids (like alkanes) on perfectly smooth and ideally homogeneous heaters where a thin liquid film covers at equilibrium the solid at room temperature. According to Derjaguin and Churaev (1974), at larger temperatures but still at equilibrium, the liquid film may disappear in the case of water. Rednikov and Colinet (2011) showed theoretically that for finite values of the Young contact angle but in the presence of the disjoining pressure $\sim h^{-3}$, the continuous film solution becomes unstable starting from some (small) values of superheating of the solid. The stable equilibrium solution is wedgelike; the dry solid appears in spite of the disjoining pressure divergence at $h \rightarrow 0$. As a result, in most practically important systems (water on metal heaters) and temperatures, the substrate surface is partially dry at equilibrium and the dependence of the bubble dynamics on the wetting properties (characterized by the equilibrium contact angle) is essential.

From the point of view of the numerical CL modeling, the continuous film approach is a priori less convenient than the partial wetting approach for several reasons. (i) The former approach requires meshing of the infinite film, which is absent in the latter. (ii) To implement the former approach one absolutely needs to introduce the disjoining pressure function, the shape of which is highly uncertain for the fluid-substrate combinations and temperature ranges 
of interest. In the latter approach the introduction of the disjoining pressure is not mandatory [although possible; see Ajaev et al. (2010); Janeček and Nikolayev (2013)], the solid-fluid interaction being accounted for by the relatively easily measurable equilibrium contact angle.

The continuous film models are popular because the presence of film relaxes the consequences of the CL geometric singularity in both the hydrodynamic and thermal problems. The hydrodynamic CL singularity in the partial wetting case is solved usually by introducing the hydrodynamic slip length $l_{s}$ (Anderson and Davis, 1995; Nikolayev, 2010; Oron et al., 1997). It has been shown by us (Janeček and Nikolayev, 2012) that the slip length is not absolutely necessary for the description of evaporation. The Kelvin effect (dependence of saturation temperature on interface curvature) alone relaxes the singularity. The slip length is however often more important than the characteristic Kelvin length (Janeček et al., 2013) and for this reason is considered below as the main microscopic length scale. Each of these effects has some impact on $\theta_{a p p}$ and for this reason both of them are accounted for. As a consequence, $\theta_{a p p}$ is only weakly dependent on each separate parameter like slip length, gas-liquid interfacial resistance $R^{i}$, or Marangoni effect, which means that the model is robust.

As in the studies cited above, we adopt the "one-sided" description, according to which the vapor pressure is assumed to be spatially homogeneous. This assumption is justified by the smallness of both density and viscosity of the vapor. The relation for the liquid-vapor interface temperature

$$
T^{i}=T_{\text {sat }}\left[1+\frac{\Delta p}{H \rho_{L}}+\frac{J^{2}}{2 H \rho_{V}^{2}}\right]+R^{i} q_{L}^{i}
$$

is based on linearized Clausius-Clapeyron formula enhanced for the interfacial resistance $R^{i}$ (Anderson and Davis, 1995; Nikolayev, 2010; Stephan and Hammer, 1994) which accounts for the molecular kinetics effects. The vapor recoil contribution (the third term) has been derived in Anderson et al. (2007).

The lubrication theory accounts for the Navier slip boundary condition (Nikolayev, 2010) at the solid wall $(y=0)$ and the Marangoni effect $d \sigma / d s=-\gamma d T^{i} / d s$ at the liquid-vapor interface, where $\gamma=-d \sigma / d T$. Together with the liquid layer height $h$ (conventional in the lubrication approximation) we operate with the arc length $\delta=r \phi$ related to $h=r \sin \phi$ (see Fig. 2) via the expression

$$
h=\delta \frac{\sin \phi}{\phi} .
$$

The main idea of hydrodynamic approach is based on the fluid flow in a wedge with a sink localized at the CL (see the Appendix). Such an approximation is justified for a strongly curved meniscus with a high slope because the evaporation is strongly localized at the CL. One obtains the following expression for the volume flux $U(s)$ flowing through the vertical cross section of liquid film confined between the heated substrate and the gas-liquid interface,

$$
\mu G(\phi) U=\frac{d \sigma}{d s}\left(\frac{\delta^{2}}{2}+\delta l_{s}\right)+\left(\frac{\delta^{3}}{3}+\delta^{2} l_{s}\right) \frac{d \Delta p}{d s},
$$

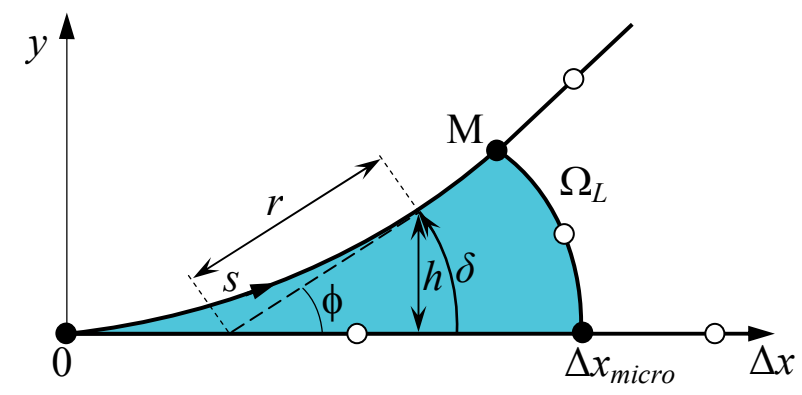

FIG. 2: Detailed sketch of the (highlighted) liquid part of microregion. The BEM nodes are shown with open circles. The arc length $\delta$, meniscus height $h$, local wedge coordinate $r$ and local slope angle $\phi$ are shown for the same point. $\theta_{a p p}$ is associated with $\phi$ at the point $M$. 
where the identity $d \Delta p / d s=-d p_{L} / d s$ is used and the CL is assumed to be immobile (the flow generated by the CL motion is thus neglected). Apart from the replacement of $h$ by $\delta$ and $x$ by $s$, Eq. (9) differs from the standard lubrication approach (Oron et al., 1997) by a correction factor $G(\phi) . G$ comes from the generalization (Mathieu, 2003) of lubrication approximation for high interface slopes, see the Appendix; $G(\phi) \rightarrow 1$ for small $\phi$ so that such an approach reduces to the standard lubrication theory when $\theta_{a p p}$ is small.

By using the fluid mass conservation law, $U$ can also be expressed via the mass evaporation flux $J$ at the gas-liquid interface (assumed positive at evaporation),

$$
U=-\frac{1}{\rho_{L}} \int_{0}^{s} J d s
$$

which can be rewritten as

$$
\frac{d U}{d s}=-\frac{J}{\rho_{L}}
$$

where $J$ is obtained below from the heat transfer part of the microregion problem.

The gas-liquid interface shape is determined from the microregion counterpart to Eq. (6)

$$
\sigma \frac{d \phi}{d s}=\Delta p+p_{r}
$$

where $p_{r}=J^{2} \rho_{V}^{-1}$ is the vapor recoil pressure; the gravity effect is negligible and $\Delta p$ varies along the vapor-liquid interface because of the liquid flow. The $h$ differential equation reads

$$
\frac{d h}{d s}=\sin \phi .
$$

The heat conduction in microregion is considered to be stationary due to the small thermal inertia of the thin liquid wedge. The temperature distribution is defined to be linear along the arc length $\delta$ (see Fig. 2) as suggested by the rigorous thermal analysis of straight wedge (Anderson and Davis, 1994),

$$
q_{L}^{i}=k_{L} \frac{T_{\text {sat }}+\Delta T-T^{i}}{\delta} .
$$

Like Stephan and Hammer (1994); Wayner et al. (1976), and Son et al. (1999), we neglect the variation of heater temperature in the microregion; $\Delta T$ is thus a parameter. As mentioned above, its value is matched to the macroregion problem.

Since the vapor heat conductivity is neglected, the interfacial heat balance reads $q_{L}^{i}=H J$. By combining it with Eqs. (8) and (14), one obtains the equation

$$
J=\frac{\Delta T-\Delta p T_{\text {sat }} /\left(H \rho_{L}\right)}{H\left(R^{i}+h / k_{L}\right)}-\frac{T_{\text {sat }}}{2 H^{4} \rho_{V}^{2}} \frac{\left[\Delta T-\Delta p T_{\text {sat }} /\left(H \rho_{L}\right)\right]^{2}}{\left(R^{i}+h / k_{L}\right)^{3}},
$$

under an assumption that the vapor recoil term in Eq. (8) is small (which has been verified a posteriori). The last equation closes the set $(9,11-13)$ for four unknown functions of $s: h, \phi, \Delta p$, and $U$. This set of ordinary differential equations is of the fourth order and requires thus four boundary conditions. In the partial wetting case considered here, two of them are geometrical constraints defined at the CL,

$$
h(s \rightarrow 0)=0, \quad \phi(s \rightarrow 0)=\theta_{\text {micro }} .
$$

The third is the matching of the microregion pressure jump at $s=s_{M}$ with its macroregion value, cf. Fig. 1(b). By using the fact that the pressure jump in macroregion $\Delta p_{\text {macro }} \sim \sigma / R$ is negligibly small with respect to its value in the vicinity of $\mathrm{CL}$, the condition

$$
\Delta p\left(s_{M}\right)=0
$$


is applied. The microregion size $\Delta x_{\text {micro }} \simeq s_{M}$ (cf. Fig. 2) is determined by the criterion that its influence on the apparent contact angle $\theta_{a p p}=\arctan u\left(s_{M}\right)$ is negligible (within the given precision). This criterion can always be satisfied because the the meniscus slope tends to $\theta_{a p p}$ as $s \rightarrow \infty$.

The fourth boundary condition

$$
\Delta p(s \rightarrow 0)=\frac{H \rho_{L} \Delta T}{T_{\text {sat }}}
$$

can be derived from Eq. (15) and the equation $J(s \rightarrow 0)=0$ (Janeček and Nikolayev, 2012), valid when the flow appearing because of the CL motion is neglected.

\subsection{Coupling of Micro and Macro Regions}

The micro and macro regions are coupled by matching the following parameters:

i. Interface slope $\left(\theta_{\text {app }}\right)$ at the microregion-macroregion junction of the vapor-liquid interface.

ii. Temperature at the part of the boundary $\Omega_{L}$ with the microregion, cf. Fig. 2. The macroregion node temperature is made equal to $0.5\left[T_{M}+T_{L}\left(\Delta x_{m i c r o}, y=0\right)\right]$ calculated in the microregion model. The heat flux cannot be matched at $\Omega_{L}$ because the asymptotic nature of the microregion treatment requires it to be fixed to zero. This mismatch appears also because of different spatial dimensions of micro (1D) and macro (2D) problems. However the error introduced by the mismatch is very small as the macroregion flux over $\Omega_{L}$ is verified to be much smaller than $Q_{\text {micro }} / \Delta x_{\text {micro }}$. To preserve the energy conservation, the flux contribution is included in Eq. (28) below.

iii. Superheating $\Delta T$ of the microregion part of the solid-liquid macroregion boundary, cf. Eq. (15) and Eq. (26) below.

iv. Heat power $Q_{\text {micro }}$ taken from the solid to the microregion through its solid-liquid boundary. Within the macroregion model, $Q_{\text {micro }}$ is the integral of $q_{S}$ over it. Since this part is implemented as a single BEM element of the length $\Delta x_{\text {micro }}, Q_{\text {micro }}$ is simply the node $q_{S}$ value times $\Delta x_{m i c r o}$. This condition corresponds to Eq. (27) below.

$Q_{m i c r o}$ is the total heat supplied from the microregion to the vapor-liquid interface,

$$
Q_{\text {micro }}=\int_{s=0}^{s_{M}} q_{L}^{i}(s) d s
$$

This value is important to the macroregion heat transfer problem for two reasons. First, its contribution to the bubble volume is not negligible, cf. Eq. (5). Second, as mentioned above, this value defines the heat flux boundary condition at the microregion part of the solid-liquid interface. These quantities are important to be matched accurately between both regions by an iterative procedure. Since $\theta_{a p p}$ and $Q_{m i c r o}$ depend only on $\Delta T$ (besides the fixed system parameters), it is possible to tabulate them and use a fitting procedure instead of recalculating them at each iteration.

Note that the coupling proposed here is more rigorous than that used by other authors, e.g., Kunkelmann and Stephan (2009), where the microregion is not considered as a separate cell but as a subgrid model for the macroscopic simulation domain. Only two parameters $\left(\theta_{a p p}\right.$ and $\left.Q_{m i c r o}\right)$ were coupled in their work. The difference of $Q_{m i c r o}$ and the calculated (within macroscopic simulation) energy balance was used as an internal to this cell source of energy. As a result, the boundary conditions were less rigorous, in particular that for the heat and mass exchange at the solidliquid and liquid-vapor microregion interfaces which turned out to be homogeneously distributed over the cell area much larger than that of the actual microregion. 


\section{PROBLEM SOLVING}

\subsection{Macroregion}

By introducing fixed scales for time ( $\Delta t$, time step), length $\left(R_{0}\right)$, heat flux $\left(q_{0}\right)$, and pressure $\left(\sigma / R_{0}\right)$, all other variables can be made dimensionless. The dimensionless temperature is $\psi=\left(T_{S, L}-T_{S, L}^{i n f}\right) k_{L} /\left(q_{0} R_{0}\right)$, where $T_{S, L}^{i n f}=$ $T_{S, L}(x \rightarrow \infty, y, t)$. The values at infinity are subtracted to eliminate the necessity of integration over the domain boundaries situated at infinity. The dimensionless heat transfer problem statement is (the tilde means the corresponding dimensionless quantity)

$$
\begin{aligned}
\frac{\partial \psi_{L, S}}{\partial t} & =\mathrm{Fo}_{L, S} \nabla^{2} \psi_{L, S}, \\
\left.\psi_{L, S}\right|_{t=0} & =0, \\
\left.\psi_{L}\right|_{\Omega_{i}} & =-\tilde{T}_{L}^{i n f}(\tilde{y}, \tilde{t}), \\
\left.\zeta_{L}\right|_{\Omega_{w}} & =\left.\zeta_{S}\right|_{\Omega_{w}}, \\
\left.\zeta_{L}\right|_{\Omega_{d}} & =-1,
\end{aligned}
$$

where $\zeta_{L}=\partial \psi_{L} / \partial \vec{n}, \zeta_{S}=k_{S} / k_{L} \partial \psi_{S} / \partial \vec{n}$, and

$$
\tilde{T}_{L}^{i n f}(\tilde{y}, \tilde{t})=2 \sqrt{\frac{\mathrm{Fo}_{L} \tilde{t}}{\pi}} \exp \left(-\frac{\tilde{y}^{2}}{4 \mathrm{Fo}_{L} \tilde{t}}\right)-\tilde{y} \operatorname{erfc}\left(\frac{\tilde{y}}{2 \sqrt{\mathrm{Fo}_{L} \tilde{t}}}\right) .
$$

The boundary conditions at the boundaries with microregion (cf. Section 2.4) are defined as follows:

$$
\begin{aligned}
\left.\psi_{L}\right|_{\Omega_{L}} & =\Delta \tilde{T} \tilde{y} / \tilde{y}_{M}-\tilde{T}_{L}^{i n f}(\tilde{y}, \tilde{t}), \\
\left.\zeta_{S}\right|_{y=0} & =\tilde{Q}_{\text {micro }} / \Delta \tilde{x}_{\text {micro }}-1 .
\end{aligned}
$$

The dimensionless bubble volume equation reads

$$
\frac{d \tilde{V}}{d \tilde{t}}=\mathrm{Fo}_{L} \mathrm{Ja}\left[\tilde{Q}_{\text {micro }}+\int_{\left(\Omega_{i} \cup \Omega_{L}\right)}\left(\zeta_{\text {inf }}-\zeta_{L}\right) d \Omega\right],
$$

where $\zeta_{\text {inf }}=n^{y} \operatorname{erfc}\left[\tilde{y} /\left(2 \sqrt{\mathrm{Fo}_{L} \tilde{t}}\right)\right]$.

By following Nikolayev et al. (2001), the bubble shape equation (6) is written in the parametric form by choosing $s$ as an independent variable, see Fig. 1(a). The dimensionless coordinates $(\tilde{x}, \tilde{y})$ for a given point on the bubble interface are then functions of $\xi=s / L$ that vary along the bubble half-contour from 0 to $1 ; \xi=0$ and $\xi=1$ correspond to the point $\mathrm{M}$ of matching of the micro and macro regions and to the topmost point of the bubble, respectively. Equation (6) is thus equivalent to the set of ordinary differential equations

$$
\begin{aligned}
& \frac{d \bar{x}}{d \xi}=\cos \phi, \\
& \frac{d \bar{y}}{d \xi}=\sin \phi, \\
& \frac{d \phi}{d \xi}=\tilde{L}\left(\Delta \tilde{p}_{\text {macro }}+\text { Bo } \tilde{y}\right),
\end{aligned}
$$

where the rescaled with $\tilde{L}$ variables $\bar{x}=\tilde{x} / \tilde{L}, \bar{y}=\tilde{y} / \tilde{L}$ are introduced. The boundary conditions for Eqs. (29)-(31) are

$$
\begin{aligned}
& \left.\tilde{x}\right|_{\xi=1}=0,\left.\quad \phi\right|_{\xi=1}=\pi, \\
& \left.\tilde{y}\right|_{\xi=0}=0,\left.\quad \phi\right|_{\xi=0}=\theta_{\text {app }} .
\end{aligned}
$$


We checked that the error introduced by the replacement of $\tilde{y}_{M}$ by zero in the third boundary condition is negligible. Only three boundary conditions are necessary to solve Eqs. (29)-(31). The fourth serves to find the unknown length $L$ via an iteration procedure. The value of $\Delta \tilde{p}_{\text {macro }}$ is independent of $\xi$ and is determined with the help of the the $\tilde{V}_{\text {macro }} \approx \tilde{V}$ value known from Eq. (28) as follows.

The equation for $\tilde{V}_{\text {macro }}$ is slightly modified with respect to that of Nikolayev et al. (2001). By using the identity $\operatorname{div}\left(x \vec{e}^{x}\right)=1$ (which is evident corollary of the divergence definition), the bubble volume can be expressed as

$$
V=\int_{\left(V_{\text {macro }}\right)} d V=\int_{\left(V_{\text {macro }}\right)} \operatorname{div}\left(x \vec{e}^{x}\right) d V=-\int_{\left(\Omega_{i} \cup \Omega_{d}\right)} x n^{x} d \Omega .
$$

The last equality is obtained with the Gauss integral theorem. Since $n^{x}=0$ at $\Omega_{d}$,

$$
\tilde{V}=\tilde{L}^{2} \int_{0}^{1} \bar{x} \bar{y}^{\prime} d \xi
$$

To obtain $\Delta \tilde{p}_{\text {macro }}$, the direct application of Eq. (34) would require an additional iteration loop. To avoid it, we follow an idea of Polevikov (2001). By integrating Eq. (31) along the bubble contour, the pressure jump is obtained directly,

$$
\Delta \tilde{p}_{\text {macro }}=\frac{\pi-\theta_{a p p}}{\tilde{L}}-\text { Bo } \int_{0}^{1} \tilde{y} d \xi
$$

where the bubble half-length is expressed with Eq. (34).

The macroscopic heat transfer problem (20)-(27) is solved similarly to Nikolayev et al. (2001) by the constant element BEM (Nikolayev and Beysens, 2002) well suitable to the free interface problems. Due to the vanishing values of $\psi, \zeta$ at infinity, one does not need to integrate over the liquid and solid boundaries situated at infinity. The meshing of the sub-contours $\Omega_{w}, \Omega_{d}$, and $\Omega_{i}$ [cf. Fig. 1(a)] follows the exponential law with the smallest element size $\left(\approx \Delta x_{\text {micro }} \theta_{\text {app }}\right)$ located in the vicinity of the boundary $\Omega_{L}$ (cf. Fig. 2 ).

In principle, the boundary $\Omega_{w}$ continues to infinity. In the numerical treatment, a finite size has to be chosen. One can verify that growing vapor bubble perturbs the temperature field described by $T_{S, L}(x \rightarrow \infty, y, t)$ only locally and the size of the perturbed domain scales as the thermal diffusion length. The size of $\Omega_{w}$ may thus be bounded by the value $4 \sqrt{\alpha_{S} t}$ based on the largest of the thermal diffusivities. We verified that the increase of $\Omega_{w}$ beyond this value does not influence the results.

Both the part of the solid-liquid boundary whithin the microregion and $\Omega_{L}$ [cf. Fig. 1(b)] are taken as single elements.

\subsection{Microregion}

Characteristic physical scales in the microregion differ strongly from those in the macroregion. Other scales thus need to be used to make the quantities dimensionless. We use $l_{s}, \sigma / l_{s}$, and $\Delta T$ as the characteristic scales for lengths, pressures, and temperatures. The hat signifies the corresponding reduced quantities. The dimensionless counterparts of the equations of Section 2.3 are not reproduced here because they are quite cumbersome; see Janeček (2012) for more details. The smallness of $h$ in the vicinity (and at) the CL is at the origin of numerical difficulties in the microregion modeling. The smallness of coefficients at the derivatives in Eq. (9) leads to the poor determination of the matrix (i.e., smallness of its diagonal elements) generated after discretization and causes numerical instabilities. In order to avoid this difficulty, the following change of variables (Janeček and Nikolayev, 2012; Nikolayev, 2010) is applied:

$$
\begin{aligned}
& \hat{s}=\exp (\eta), \\
& \hat{\delta}=\chi \exp (\eta) .
\end{aligned}
$$


The problem is solved for $\chi$ and $\Delta \hat{p}$ as functions of $\eta$. The point $\hat{s}=0$ corresponds to $\eta \rightarrow-\infty$ and a minimal value $\eta_{\min }$ of $\eta$ needs to be chosen in such a way that $\delta\left(\eta_{\min }\right)$ is smaller than the smallest length scale of the microregion problem. The value $\eta_{\min }=-9$ is found to be sufficiently small throughout the calculation. The size of the domain $\Delta x_{\text {micro }}=1 \mu \mathrm{m}$ is sufficiently large to calculate $\theta_{\text {app }}$ within $1 \%$ accuracy.

An equidistant mesh of 250 elements is used for the domain $\eta \in\left(\eta_{\text {min }}, \ln \Delta \hat{x}_{\text {micro }}\right)$. In the $s$ representation, such a mesh is increasingly dense when $s \rightarrow 0$. The nonlinearity is treated with the iteration method, where nonlinear terms are replaced by their counterparts from the previous iteration. The degree of nonlinearity grows with the heat load. However, even for the highest superheating, the convergence is obtained within several iterations.

The simulation has been carried out for water at $10 \mathrm{MPa}$ pressure on the stainless steel heater [see Nikolayev (2010); Nikolayev et al. (2001) for their parameters], and for FC-72 (Raj et al., 2012). It is assumed that the surface is smooth and chemically homogeneous as attainable in the state-of-the-art experiments at the partial wetting conditions; this corresponds (Lauga et al., 2007) to the typical value of $l_{s}=10 \mathrm{~nm}$. The same slip length value is used for both water and FC-72; the $R^{i}$ is calculated from an equation provided in Stephan and Hammer (1994) with accommodation coefficient equal to 1 .

The apparent contact angle $\theta_{a p p}$ and the integrated heat flux $Q_{\text {micro }}$ obtained from the microregion numerical model are plotted vs. wall superheating in Fig. 3 for water at $10 \mathrm{MPa}$ (which is the fluid that we consider hereafter). One has to put a reserve on the microregion results for high slopes of the interface $\left(\theta_{\text {app }}>40^{\circ}\right)$ because of the lubrication approximation in spite of its extension. The results are however coherent with those of another research group (Kunkelmann and Stephan, 2009; Raj et al., 2012) obtained for FC-72 at $\Delta T<15 \mathrm{~K}$. The agreement is excellent with the theoretical data with no fitting applied. The experimental data for $\theta_{a p p}$ (Raj et al., 2012) are compared also and show reasonably good agreement. The value $\theta_{\text {micro }}=8^{\circ}$ has been chosen because it is the equilibrium contact angle value experimentally measured by Raj et al. (2012). Note that unlike earlier studies, we need the data for larger values of wall superheating to treat $\mathrm{BC}$.

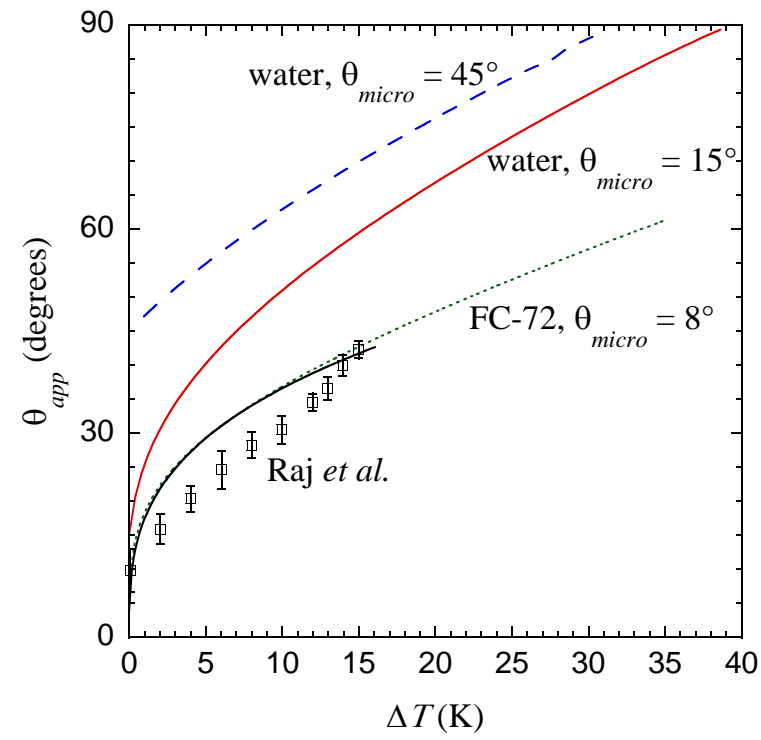

(a)

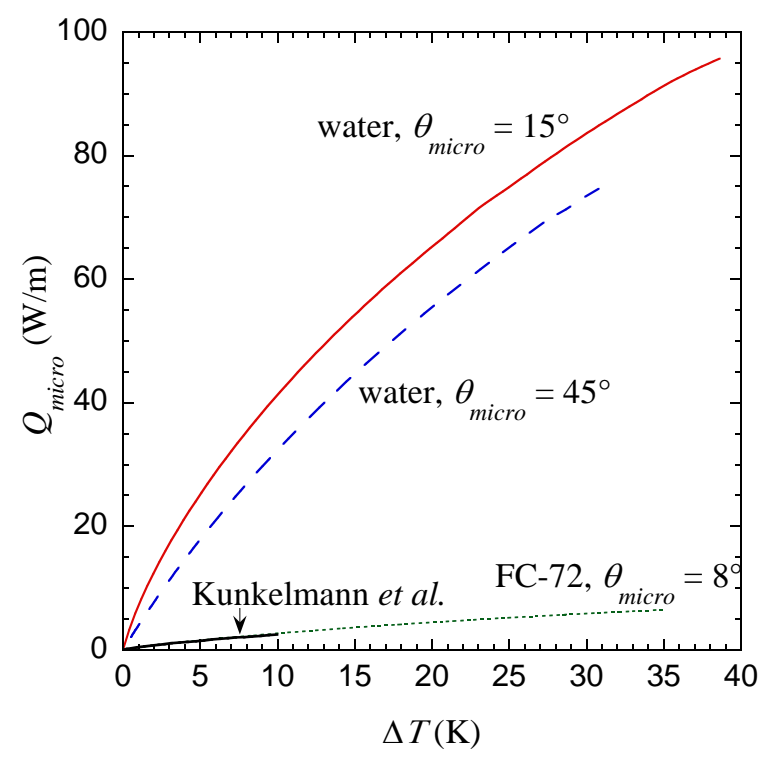

(b)

FIG. 3: Apparent contact angle $\theta_{a p p}$ and $Q_{\text {micro }}$ calculated for water at $10 \mathrm{MPa}$ and two different $\theta_{\text {micro }}$ values used in the bubble growth simulations. Curves calculated for FC-72 at $0.04 \mathrm{MPa}$ and $\theta_{\text {micro }}=8^{\circ}$ (dotted lines) are shown for comparison with the theoretical results obtained for complete wetting by another group (Kunkelmann and Stephan, 2009; Raj et al., 2012), black solid lines. (a) Apparent contact angle. The experimental data of Raj et al. (2012) (where $\theta_{\text {micro }}=8^{\circ}$ ) are also shown. (b) Total heat $Q_{\text {micro }}$ transferred through the microregion. 


\subsection{Algorithm}

The problem is nonlinear because of nonlinear terms and especially due to the free boundary. The following iteration algorithm is needed to determine the bubble shape and the thermal field at each time step:

1. The interface bubble shape, the bubble interface velocity (needed for the BEM calculation, [see Nikolayev et al. (2001) for details] and $Q_{m i c r o}$ are guessed to be the same as at the previous time step;

2. Discretization of the contours $\Omega_{w}, \Omega_{d}$, and $\Omega_{i}$ is performed;

3. Temperatures and fluxes on the contours $\Omega_{w}, \Omega_{d}$, and $\Omega_{i}$ are found by solving Eqs. (20)-(27) by BEM for $\psi_{L, S}$, $\zeta_{L, S} ;$ the value of $\Delta T$ is obtained;

4. Based on $\Delta T$, the microregion calculation is performed and new values for $\tilde{Q}_{\text {micro }}, \theta_{\text {app }}$ are computed;

5. New $\tilde{V}$ is calculated from Eq. (28) with the trapezoidal rule;

6. Bubble shape is determined using the values of $\tilde{y}$ and $\tilde{L}$ from previous iteration. First, $\Delta \tilde{p}_{\text {macro }}$ is determined with Eq. (35). Next, Eq. (31) is solved for $\phi$. The latter is used to integrate Eqs. (29), (30). Finally, $\tilde{L}$ is updated with Eq. (34), and $\tilde{x}=\tilde{L} \bar{x}, \tilde{y}=\tilde{L} \bar{y}$ are recalculated;

7. The interface velocity is calculated, and steps $2-7$ are repeated until the desired accuracy of $\theta_{a p p}, R_{d}$ and $V$ is attained.

Note that CL position is determined in the step 6: CL is a part of the bubble surface.

As a rule, four iterations give $0.2 \%$ accuracy. In order to reduce the calculation time at step 4 , the precalculated values of $\theta_{\text {app }}(\Delta T)$ and $Q_{\text {micro }}(\Delta T)$ are interpolated by cubic splines. By performing full micro-macro coupling procedure, we checked that this shortcut does not influence the results. There is one more reason in using the tabulation of the microregion values for parameters $Q_{\text {micro }}$ and $\theta_{a p p}$. The described microregion model can provide the results for $\theta_{\text {app }} \leq 89.8^{\circ}$. Both $\theta_{a p p}$ and $Q_{\text {micro }}$ are extrapolated to $90^{\circ}$ with a power law.

We verified that increasing the time step from 1 to $50 \mathrm{~ms}$ very slightly influenced the calculation accuracy without impacting the numerical stability. This is an intrinsic property of BEM. Typically the time step $\Delta t=20 \mu \mathrm{s}$ was used.

\section{RESULTS AND DISCUSSION}

Depending on the heat load, two bubble growth regimes can be distinguished. At low heat loads [Fig. 4(a)], the bubble volume increases until the buoyancy force becomes equal to the capillary adhesion force, which corresponds to the bubble departure. In the beginning of growth, the dry spot increases. At some point of growth, $R_{d}$ attains its maximum, while both the bubble volume and the visible from the top bubble radius $R$ keep growing [cf. Fig. $5(\mathrm{a}), 0.05 \mathrm{MW} / \mathrm{m}^{2}$ curves]. Therefore the quotient $R_{d} / R$ decreases at the end of the bubble evolution; see the $0.05 \mathrm{MW} / \mathrm{m}^{2}$ curve in Fig. 5(b). This regime is called hereafter "bubble departure regime."

At high heat loads [Fig. 4(b)], the condition $\theta_{a p p}=90^{\circ}$ is attained while the bubble is attached to the heater. This is the "bubble spreading regime" that corresponds to the $\mathrm{BC}$ triggering. It is possible that $\theta_{a p p}$ grows beyond $90^{\circ}$ in reality while the bubble remains attached. However, the present microregion model cannot describe such an event; the simulation is stopped as soon as $\theta_{a p p}$ attains $90^{\circ}$. Within the present quasistatic macroregion approach, the latter condition is equivalent to $R_{d}=R$ [cf. Section 2.2.2 and Fig. 5(b)]. The boundary between the bubble departure and bubble spreading regimes corresponds to CHF. At CHF, the conditions of bubble departure and $\theta_{a p p}=90^{\circ}$ are satisfied simultaneously; cf. the $0.1 \mathrm{MW} / \mathrm{m}^{2}$ curve in Fig. 5(b).

The value of the ratio $R_{d} / R$ at the moment of bubble departure is plotted vs. heat flux in Fig. 6. Each point of these curves corresponds to the terminal point of a temporal curve like those of Fig. 5. One can see that the ratio $R_{d}^{d e p} / R^{d e p}$ grows with the heat flux. The $\mathrm{BC}$ criterion is satisfied when $R_{d}^{d e p} / R^{d e p}$ attains unity.

The accuracy of the bubble shape calculation may be checked independently. Indeed, the departure bubble shape corresponds to the criterion $\Delta p_{\text {macro }}=0$; cf. Section 2.2.2. In addition, CHF attained at $\theta_{\text {app }}=90^{\circ}$. These two 


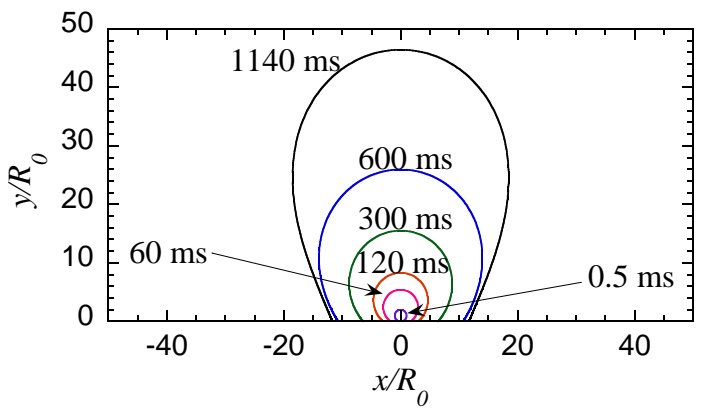

(a)

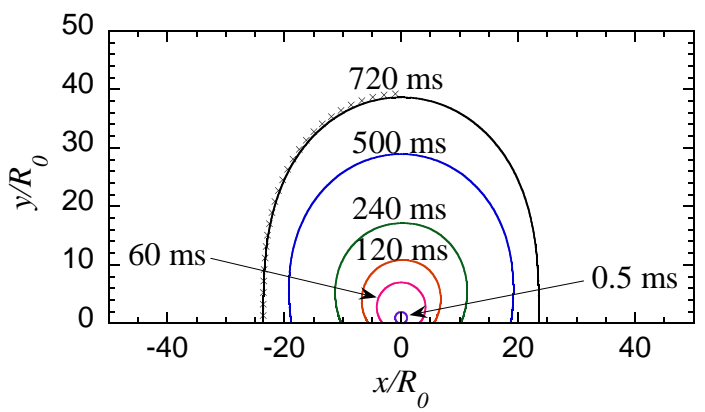

(b)

FIG. 4: Bubble shape evolution shown for $\theta_{\text {micro }}=15^{\circ}$ and $R_{0}=50 \mu \mathrm{m}$. (a) $q_{0}=0.05 \mathrm{MW} / \mathrm{m}^{2}$. The $1140 \mathrm{~ms}$ profile corresponds to the bubble departure. (b) CHF situation: $q_{0}=0.1 \mathrm{MW} / \mathrm{m}^{2}$. The $720 \mathrm{~ms}$ profile corresponds to the bubble departure. It is verified by the independent calculation (crosses) as described in the text.

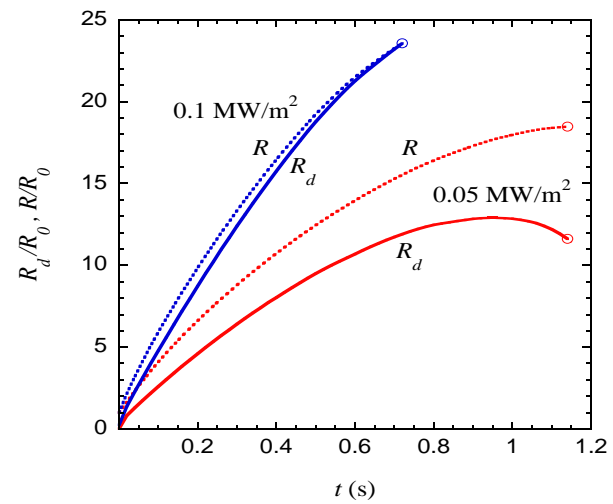

(a)

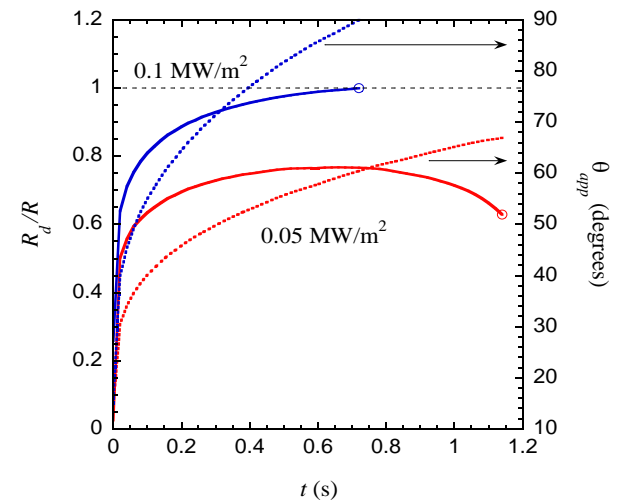

(b)

FIG. 5: The temporal evolution of (a) $R_{d}$ and $R$ (see Fig. 1 for their definition), and (b) their quotient (full lines) and $\theta_{\text {app }}$ (dotted lines) for the same parameters as Fig. 4. The terminal points (corresponding to the bubble departure) are indicated with open circles.

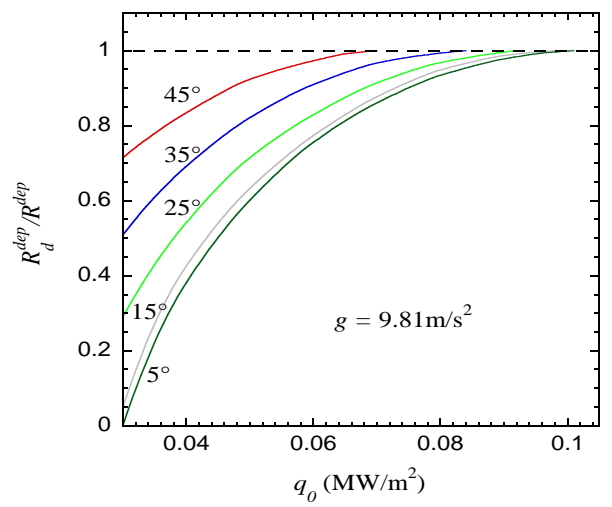

FIG. 6: The quotient of the dry spot radius and the bubble radius at the moment of bubble departure as a function of heat flux for different values of $\theta_{\text {micro }}$. The CHF is attained when $R_{d}^{d e p} / R^{d e p}$ reaches unity. 
conditions are sufficient to find the bubble shape from Eq. (6) for given $g$ independently of the heat transfer calculation. The bubble half-contour can be obtained by using two boundary conditions: $d y / d s=0$ at $x=0$ and $d x / d s=0$ at $y=0$. For the given fluid parameters and $g$ value, one obtains a unique value of $R_{d}^{d e p}=R^{d e p}$; for water at $10 \mathrm{MPa}$ and $g=9.81 \mathrm{~m} / \mathrm{s}^{2}$ this value is $1.18 \mathrm{~mm}$. The shape calculation carried out by the standard Runge-Kutta method is shown by the (half-contour) dotted line in Fig. 4(b). It nearly coincides with its counterpart calculated for the moment of departure $(t=720 \mathrm{~ms})$ with the general algorithm, which shows the simulation accuracy. It is important to account for the actual (noncircular) shape of the bubble provided by the buoyancy. If the circular shape was imposed in the simulation, an essentially different value of $1.57 \mathrm{~mm}$ would result from the force balance at departure. This is expected because under the gravity the bubble extends in the vertical direction [Fig. 4(a)] so that its size in the perpendicular direction is smaller than that calculated from the circular approximation.

In both regimes the apparent contact angle grows with time which corresponds to experimental observations (Garrabos et al., 2001; Raj et al., 2012); see Fig. 5(b).

Our simulation enables the temperature distribution along the heater surface to be rigorously calculated. It is shown in Fig. 7, which corresponds to the bubble profiles of Fig. 4. Far from the bubble, the surface temperature is described by Eq. (4). It increases in time independently of $x$ and follows the square root law. At the CL, $T_{S}=T_{\text {sat }}+\Delta T$ as shown in the insert of Fig. 7(a). The heater temperature drop at the CL is due to the intensive latent heat consumption in the microregion. Such a result agrees with experimental observations and other numerical simulations, see e.g. (Kunkelmann and Stephan, 2009; Sodtke et al., 2006). Inside the dry spot, $T_{S}$ increases with time because the heat transfer through the dry spot is blocked. However, the temperature remains smaller than the temperature far from the bubble when the dry spot is small enough (in the bubble departure regime, cf. Fig. 7(a) because of the low temperature at the CL. In the opposite case [Fig. 7(b)], the dry spot becomes to be hotter than the rest of the heater. This temperature increase leads eventually to the burnout of the heater observed during the BC.

The temperature distribution evolution inside the heater and the liquid is shown for $q_{0}$ both below and above CHF in Fig. 8. One can clearly see all the described above phenomena as well as the temperature boundary layers.

A parametric CHF study for different gravity levels and contact angles has been carried out.

The main parameter of the microregion model is the constant microscopic contact angle imposed by the surface energies of the fluid and the heater. We have studied the impact of this undoubtedly important parameter on the bubble growth, spreading and departure. Figure 6 shows the ratio $R_{d} / R$ at departure for several values of the contact angle.

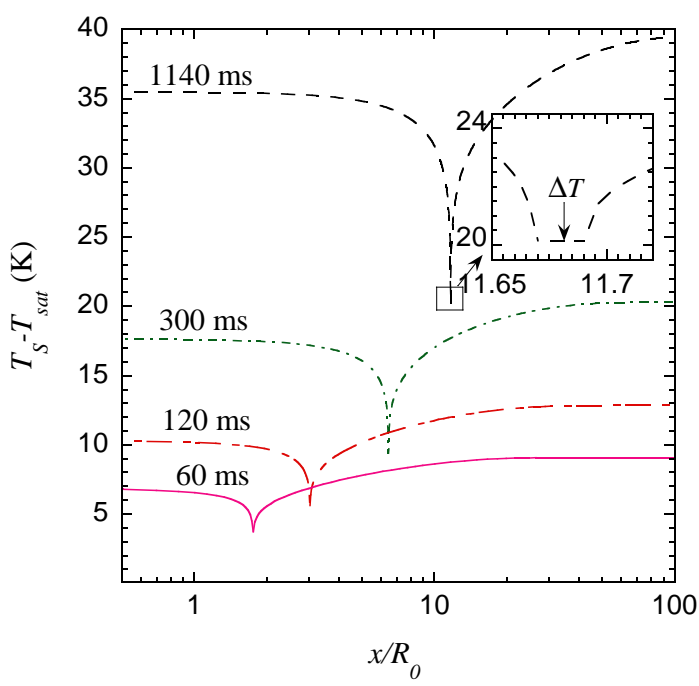

(a)

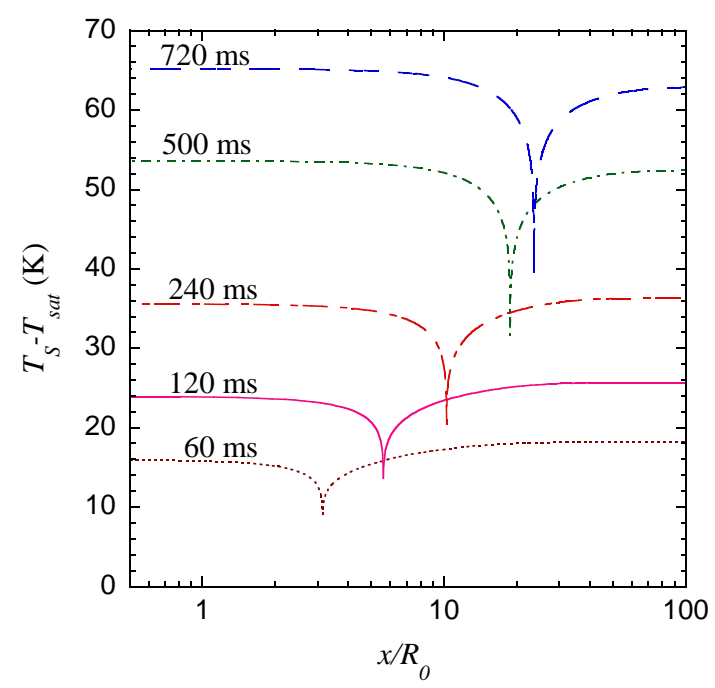

(b)

FIG. 7: Variation of the temperature along the heater surface different growth times (corresponding to those of Fig. 4) for $\theta_{\text {micro }}=15^{\circ}$ and for (a) $q_{0}=0.05 \mathrm{MW} / \mathrm{m}^{2}$, (b) $q_{0}=0.1 \mathrm{MW} / \mathrm{m}^{2}$. 


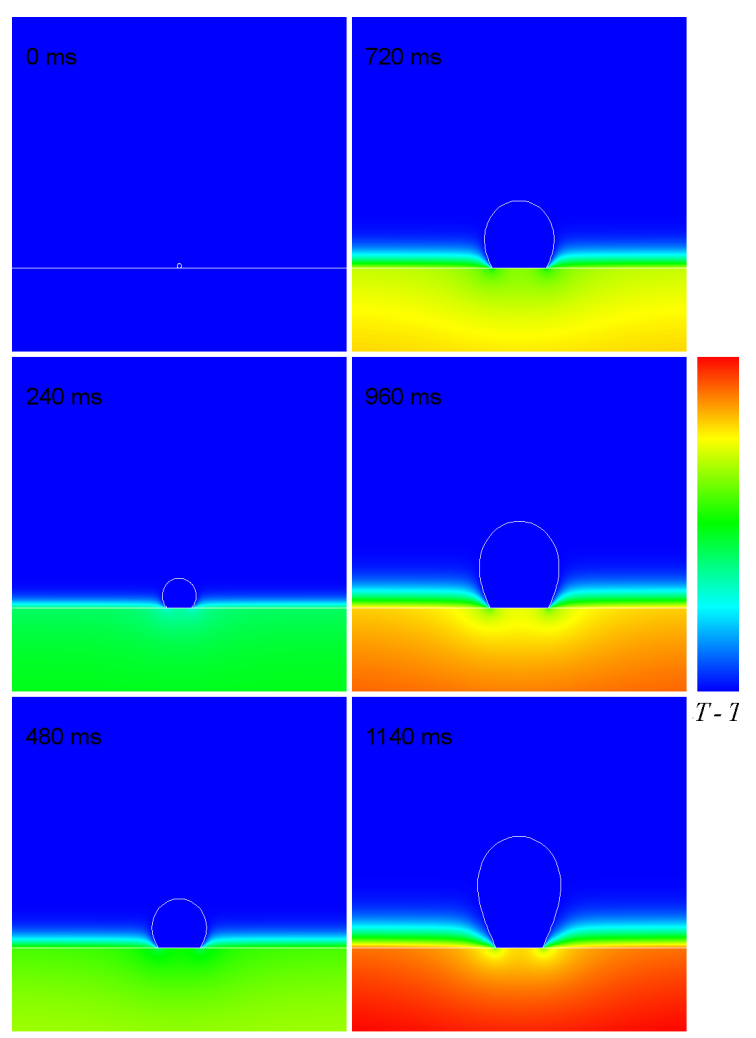

(a)

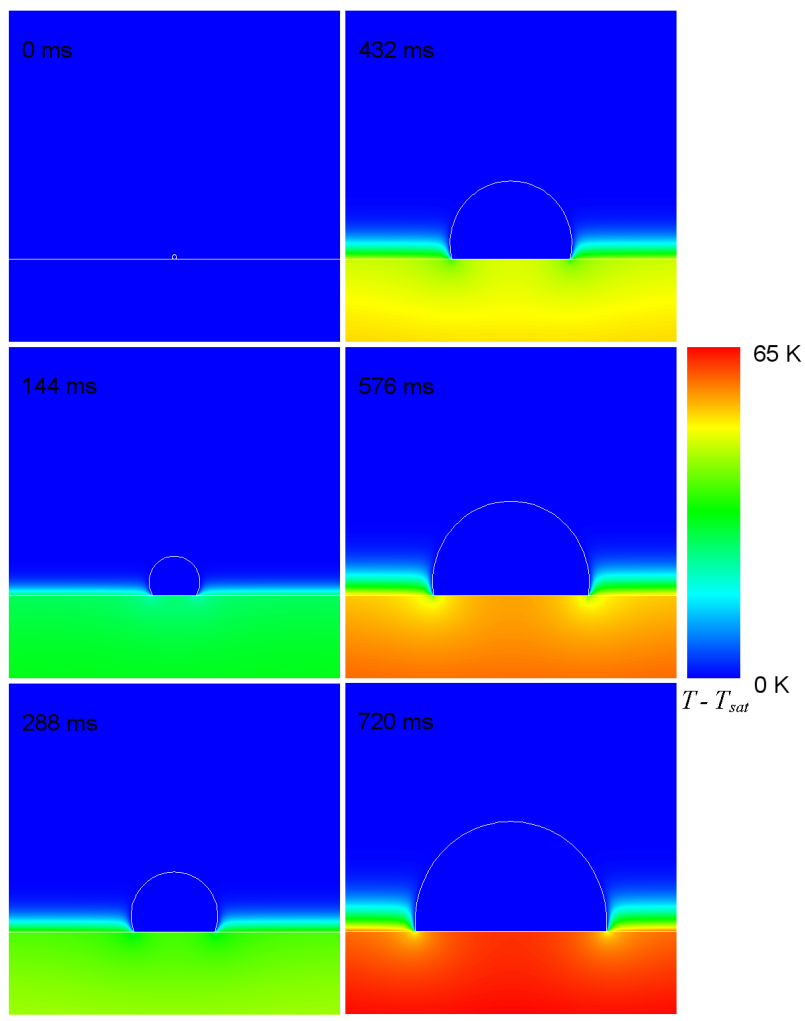

(b)

FIG. 8: Temperature field in the liquid and in the heater for six different times shown for $\theta_{\text {micro }}=15^{\circ}$; (a) $q_{0}=$ $0.05 \mathrm{MW} / \mathrm{m}^{2}$ (below CHF) and (b) $0.1 \mathrm{MW} / \mathrm{m}^{2}$ (at CHF).

The difference between the curves for $\theta_{\text {micro }}=5^{\circ}$ and $15^{\circ}$ is very small with respect to the pair $35^{\circ}$ and $45^{\circ}$ where the difference is striking. With the heat load increase, $R_{d}^{d e p} / R^{d e p}$ increases and attains unity at the CHF.

The impact of $\theta_{\text {micro }}$ on the critical heat flux is illustrated in Fig. 9 for two values of $g$. For decreasing $\theta_{\text {micro }}$, $q_{C H F}$ saturates at a value corresponding to the complete wetting case. This tendency is in agreement with the small difference between results for $\theta_{\text {micro }}=5^{\circ}$ and $15^{\circ}$ in Fig. 6 . The CHF decrease with the contact angle agrees with the experimental results (Dhir, 1998; Kandlikar, 2001). The experimental data for small contact angles usually show a somewhat steeper change than that of Fig. 9. The experimental dependencies on the contact angle should however be considered with caution because it is difficult to isolate the effect of the contact angle: other material properties often vary with its change. The large measured experimentally values of equilibrium contact angle are often due to the CL pinning on the heater surface heterogeneities [responsible for the wetting hysteresis; see Nikolayev (2005)] rather than to the surface energy effect that influences the CL dynamics considered here. The equilibrium contact angle usually remains small for clean and smooth substrates.

The impact of gravity on the critical heat flux is analyzed in Fig. 10. The CHF is an increasing function of gravity in agreement with experimental observations (Kim et al., 2002). The gravity dependence is well fitted by a power law with the exponent $\simeq 0.44$ which is larger than that predicted by Eq. (1).

To obtain all the above data on the boiling crisis within a relatively simple numerical approach, the hydrodynamic flows in the macroregion have been neglected. Their account would require the use of a full hydrodynamic simulation with an efficient management of the moving gas-liquid interfaces with phase exchange. Therefore, this work should be considered as a preparation step for such a study necessary to have the benchmark and the understanding of main 


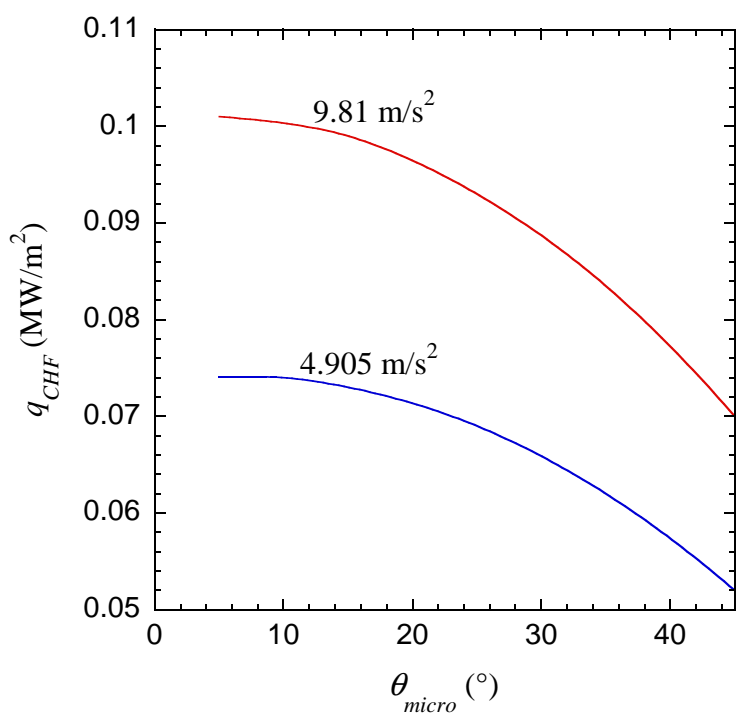

FIG. 9: Critical heat flux $q_{C H F}$ as a function of the contact angle for two different values of $g$.

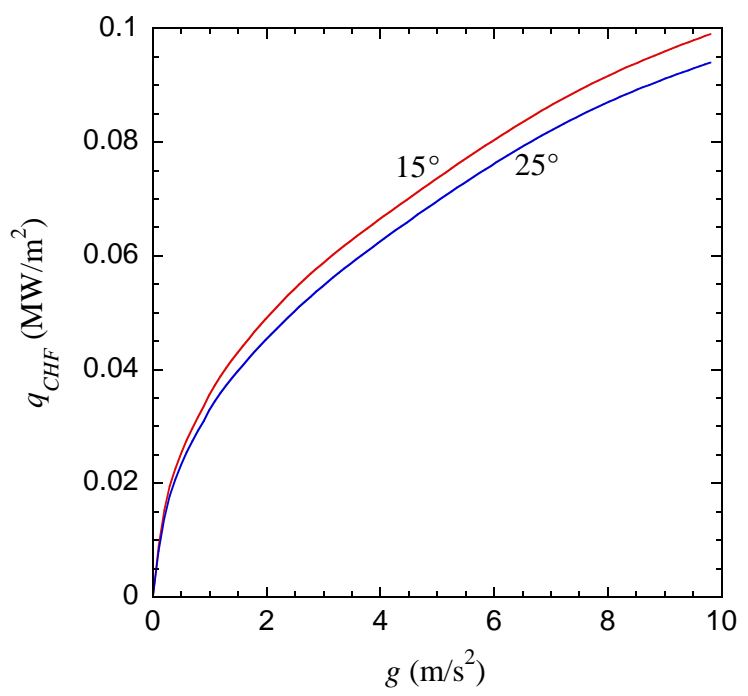

FIG. 10: Critical heat flux $q_{C H F}$ as a function of the gravity acceleration for two different contact angles.

features of the BC. It is difficult to provide a quantitative agreement with the experiment within the present simplified bubble growth model; for this reason we discussed above only the qualitative features of the BC triggering.

The boiling crisis is not an instantaneous phenomenon, it develops progressively. Once its triggering occurs, its dynamics (and thus duration) is controlled by several processes. First, it is the bubble spreading studied here. Next, the bubble interaction should be considered. The bubbles may interact both temporally and spatially. The temporal interaction manifests itself through the thermal history of a particular nucleation site that becomes more and more hot after each bubble growth and departure cycle. The spatial interaction occurs both through coalescence of neighboring bubbles that causes further extension of the dry heater area and through the heat diffusion inside the heater that controls the general heater temperature rise during BC. These processes have been discussed in a recent paper (Charignon et al., 2015). 


\section{CONCLUSIONS}

A numerical simulation of the single vapor bubble growth on a massive heater has been performed in order to study the triggering of the boiling crisis. It is shown that phenomena acting in the vicinity of the triple contact line (the microregion) have a strong impact on the vapor bubble dynamics. The microregion has been modeled for the case of partial wetting where the microscopic contact angle is an input parameter. Several quantities are determined from the microregion model, including the apparent contact angle. The latter is larger than the microscopic (static) contact angle because of evaporation. To provide a description of the apparent contact angles up to $90^{\circ}$, an extension of the conventional lubrication approximation has been used. The microregion model has been validated against the results of another group.

Two regimes of bubble growth have been identified. The first regime is the conventional bubble growth-anddeparture regime. The second, "bubble spreading" regime occurs when the wall superheating is high enough to cause a strong increase of the apparent contact angle that, in turn, causes a strong increase of the dry area under the bubble. The apparent contact angle is found to grow during the bubble growth. The $90^{\circ}$ apparent contact angle value is suggested to be an important threshold for several reasons. (i) Dry area growth causes the heater temperature rise that should lead to the increase of the apparent contact angle of neighboring bubbles. (ii) Strong adhesion to the heater caused by the large apparent contact angle should favor the coalescence of bubbles attached to the heater. (iii) Coalescence of the dry areas under the bubbles occurs during the bubble coalescence, which causes the heater temperature rise favoring the spreading of neighboring bubbles and therefore coalescence of the newly formed bubble with its neighbors. Therefore, the individual bubble spreading is proposed to be the triggering mechanism of the boiling crisis. The heat flux corresponding to $90^{\circ}$ apparent contact angle attained at the moment of bubble departure is proposed to be the CHF criterion. The CHF obtained from such a criterion via simulation has been studied as a function of gravity and of the contact angle. The results correspond at least qualitatively to the available experimental data. However the results do not seem to agree with the Zuber-Kutateladze formula since they provide a stronger dependence of CHF on gravity (i.e., a larger power law exponent) and show quite important dependence of CHF on the equilibrium contact angle. To obtain a better agreement with experiments, a more realistic model of macroregion (accounting for the fluid motion in it) needs to be implemented within a single bubble growth simulation.

\section{ACKNOWLEDGMENTS}

The financial support of ANR (project ALICE, ANR-08-BLAN-0212-03) and from CNES is acknowledged. We thank B. Mathieu for giving us access to his thesis and for fruitful discussions.

\section{REFERENCES}

Ajaev, V. S., Spreading of thin volatile liquid droplets on uniformly heated surfaces, J. Fluid Mech., vol. 528, pp. 279-296, 2005.

Ajaev, V. S., Gambaryan-Roisman, T., and Stephan, P., Static and dynamic contact angles of evaporating liquids on heated surfaces, J. Colloid Interface Sci., vol. 342, no. 2, pp. 550-558, 2010.

Anderson, D. M., Cermelli, P., Fried, E., Gurtin, M. E., and McFadden, G. B., General dynamical sharp-interface conditions for phase transformations in viscous heat-conducting fluids, J. Fluid Mech., vol. 581, pp. 323-370, 2007.

Anderson, D. M. and Davis, S. H., Local fluid and heat flow near contact lines, J. Fluid Mech., vol. 268, pp. 231-265, 1994.

Anderson, D. M. and Davis, S. H., The spreading of volatile liquid droplets on heated surfaces, Phys. Fluids, vol. 7, pp. 248-265, 1995.

Boender, W., Chesters, A. K., and van der Zanden, A. J. J., An approximate analytical solution of the hydrodynamic problem associated with an advancing liquid-gas contact line, Int. J. Multiphase Flow, vol. 17, pp. 661-676, 1991.

Bricard, P., Péturaud, P., and Delhaye, J.-M., Understanding and modeling DNB in forced convective boiling: Modeling of a mechanism based on nucleation site dryout, Multiphase Sci. Techn., vol. 9, no. 4, pp. 329-379, 1997.

Buyevich, Y. A., Towards a unified theory of pool boiling - the case of ideally smooth heated wall, Int. J. Fluid Mech. Res., vol. 26, pp. 189-223, 1999. 
Charignon, T., Lloveras, P., Chatain, D., Truskinovsky, L., Vives, E., Beysens, D., and Nikolayev, V. S., Criticality in the slowed down boiling crisis at zero gravity, Phys. Rev. E, vol. 91, 053007, 2015.

Chatpun, S., Watanabe, M., and Shoji, M., Nucleation site interaction in pool nucleate boiling on a heated surface with triple artificial cavities, Int. J. Heat Mass Transfer, vol. 47, no. 14-16, pp. 3583-3587, 2004.

Chung, H. J. and No, H. C., Simultaneous visualization of dry spots and bubbles for pool boiling of R-113 on a horizontal heater, Int. J. Heat Mass Transfer, vol. 46, pp. 2239-2251, 2003.

Cooper, M. G. and Chandratilleke, T. T., Growth of diffusion-controlled vapour bubbles at a wall in a known temperature gradient, Int. J. Heat Mass Transfer, vol. 24, no. 9, pp. 1475-1492, 1981.

Delon, G., Fermigier, M., Snoeijer, J. H., and Andreotti, B., Relaxation of a dewetting contact line, Part 2: Experiments, J. Fluid Mech., vol. 604, pp. 55-75, 2008.

Derjaguin, B. V. and Churaev, N. V., Structural component of disjoining pressure, J. Colloid Interface Sci., vol. 49, no. 2, pp. 249-255, 1974.

Dhir, V. K., Boiling heat transfer, Ann. Rev. Fluid Mech., vol. 30, pp. 365-401, 1998.

Garrabos, Y., Lecoutre-Chabot, C., Hegseth, J., Nikolayev, V. S., Beysens, D., and Delville, J.-P., Gas spreading on a heated wall wetted by liquid, Phys. Rev. E, vol. 64, no. 5, 051602, 2001.

Golobic, I., Petkovsek, J., Gjerkes, H., and Kenning, D. B. R., Horizontal chain coalescence of bubbles in saturated pool boiling on a thin foil, Int. J. Heat Mass Transfer, vol. 54, no. 25-26, pp. 5517-5526, 2011.

Gong, S., Ma, W., and Dinh, T.-N., An experimental study of rupture dynamics of evaporating liquid films on different heater surfaces, Int. J. Heat Mass Transfer, vol. 54, no. 7-8, pp. 1538-1547, 2011.

Gong, S., Ma, W., and Gu, H., An experimental investigation on bubble dynamics and boiling crisis in liquid films, Int. J. Heat Mass Transfer, vol. 79, pp. 694-703, 2014.

Janeček, V., Evaporation at Microscopic Scale and at High Heat Flux, PhD Thesis, Université Pierre et Marie Curie Paris 6, 2012.

Janeček, V., Andreotti, B., Pražák, D., Bárta, T., and Nikolayev, V. S., Moving contact line of a volatile fluid, Phys. Rev. E, vol. 88, 060404, 2013

Janeček, V. and Nikolayev, V. S., Contact line singularity at partial wetting during evaporation driven by substrate heating, Europhys. Lett., vol. 100, no. 1, 14003, 2012.

Janeček, V. and Nikolayev, V. S., Apparent-contact-angle model at partial wetting and evaporation: Impact of surface forces, Phys. Rev. E, vol. 87, 012404, 2013.

Jung, J., Kim, S. J., and Kim, J., Observations of the critical heat flux process during pool boiling of FC-72, J. Heat Transfer, vol. 136, no. 4, p. 041501, 2014.

Kandlikar, S. G., A theoretical model to predict pool boiling CHF incorporating effects of contact angle and orientation, J. Heat Transfer, vol. 123, no. 6, pp. 1071-1079, 2001.

Kandlikar, S. G. and Steinke, M. E., Contact angles and interface behavior during rapid evaporation of liquid on a heated surface, Int. J. Heat Mass Transfer, vol. 45, pp. 3771-3780, 2002.

Kannengieser, O., Bergez, W., and Colin, C., Boiling on an isolated nucleation site close to CHF conditions, Proc. 15th Int. Heat Transfer Conf., Kyoto, Japan, 2014, paper IHTC15-8856.

Kenning, D. B. R. and Del Valle, V. H., Fully-developed nucleate boiling: Overlap of areas of influence and interference between bubble sites, Int. J. Heat Mass Transfer, vol. 24, no. 6, pp. 1025-1032, 1981.

Kim, J., Benton, J. F., and Wisniewski, D., Pool boiling heat transfer on small heaters: Effect of gravity and subcooling, Int. J. Heat Mass Transfer, vol. 45, no. 19, pp. 3919-3932, 2002.

Kunkelmann, C. and Stephan, P., CFD simulation of boiling flows using the volume-of-fluid method within OpenFOAM, Numer. Heat Transfer, Part A, vol. 56, no. 8, pp. 631-646, 2009.

Kutateladze, S. S., Hydromechanical model of heat transfer crisis in pool liquid boiling, J. Tech. Phys., no. 11, pp. 1389-1392, in Russian, 1950.

Lauga, E., Brenner, M. P., and Stone, H. A., Microfluidics: The no-slip boundary condition, in Springer Handbook of Experimental Fluid Dynamics, Tropea, C., Yarin, A., and Foss, J., (Eds.), Springer, New York, Ch. 19, pp. 1217-1240, 2007.

Lloveras, P., Salvat-Pujol, F., Truskinovsky, L., and Vives, E., Boiling crisis as a critical phenomenon, Phys. Rev. Lett., vol. 108, 
$215701,2012$.

Mathieu, B., Etudes Physiques, Expérimentale et Numérique des Mécanismes de Base Intervenant Dans les Écoulements Diphasiques, PhD Thesis, Polytech Marseille, Université de Provence, 2003.

Moosman, S. and Homsy, G. M., Evaporating menisci of wetting fluids, J. Colloid Interface Sci., vol. 73, no. 1, pp. 212-223, 1980.

Mukherjee, A. and Dhir, V. K., Study of lateral merger of vapor bubbles during nucleate pool boiling, J. Heat Transfer, vol. 126, no. 6, pp. 1023-1039, 2004.

Nikolayev, V. S., Dynamics and depinning of the triple contact line in the presence of periodic surface defects, J. Phys. Cond. Matt., vol. 17, no. 13, pp. 2111-2119, 2005.

Nikolayev, V. S., Dynamics of the triple contact line on a nonisothermal heater at partial wetting, Phys. Fluids, vol. 22, no. 8, 082105, 2010

Nikolayev, V. S. and Beysens, D. A., Boiling crisis and non-equilibrium drying transition, Europhys. Lett., vol. 47, no. 3, pp. 345-351, 1999.

Nikolayev, V. S. and Beysens, D. A., 2002. 2D BEM modeling of a singular thermal diffusion free boundary problem with phase change, in Boundary elements XXIV (Incorporating meshless solutions), Brebbia, C. A., Tadeu, A., and Popov, V. (Eds.), vol. 13 of Int. Series on Advances in Boundary Elements, WIT Press, Southampton, pp. 501-525, 2002.

Nikolayev, V. S., Beysens, D. A., Lagier, G.-L., and Hegseth, J., Growth of a dry spot under a vapor bubble at high heat flux and high pressure, Int. J. Heat Mass Transfer, vol. 44, no. 18, pp. 3499-3511, 2001.

Nikolayev, V. S., Chatain, D., and Beysens, D., Bubble spreading during the boiling crisis: Modeling and experimenting in microgravity, in Proc. 6th Intl. Conf. on Boiling Heat Transfer, Spoleto, Italy, 2006a.

Nikolayev, V. S., Chatain, D., Garrabos, Y., and Beysens, D., Experimental evidence of the vapor recoil mechanism in the boiling crisis, Phys. Rev. Lett., vol. 97, 184503, 2006b.

Nikolayev, V. S. and Janeček, V., Impact of the apparent contact angle on the bubble departure at boiling, Int. J. Heat Mass Transfer, vol. 55, no. 23-24, pp. 7352-7354, 2012.

Oron, A., Davis, S. H., and Bankoff, S. G., Long-scale evolution of thin liquid films, Rev. Mod. Phys., vol. 69, no. 3, pp. 931-980, 1997.

Pasamehmetoglu, K. O. and Nelson, R. A., Cavity-to-cavity interaction in nucleate boiling: the effect of heat conduction within the heater, AIChE Symp. Ser, vol. 87, no. 283, pp. 342-351, 1991.

Polevikov, K. P., Methods for numerical modeling of two-dimensional capillary surfaces, Comput. Meth. Appl. Math., vol. 4, pp. 66-93, 2001.

Raj, R., Kunkelmann, C., Stephan, P., Plawsky, J., and Kim, J., Contact line behavior for a highly wetting fluid under superheated conditions, Int. J. Heat Mass Transfer, vol. 55, no. 9-10, pp. 2664-2675, 2012.

Rednikov, A. Y. and Colinet, P., Truncated versus extended microfilms at a vapor-liquid contact line on a heated substrate, Langmuir, vol. 27, no. 5, pp. 1758-1769, 2011.

Snoeijer, J. H., Free-surface flows with large slopes: Beyond lubrication theory, Phys. Fluids, vol. 18, 021701, 2006.

Sodtke, C., Schweizer, N., Wagner, E., and Stephan, P., Experimental study of nucleate boiling heat transfer under low gravity conditions using TLCs for high resolution temperature measurements, in Proc. 6th Intl. Conf. on Boiling Heat Transfer, Spoleto, Italy, 2006.

Son, G., Dhir, V. K., and Ramanujapu, N., Dynamics and heat transfer associated with a single bubble during nucleate boiling on a horizontal surface, J. Heat Transfer, vol. 121, no. 3, pp. 623-631, 1999.

Stephan, P. and Hammer, J., A new model for nucleate boiling heat transfer, Heat Mass Transfer, vol. 30, no. 2, pp. 119-125, 1994.

Theofanous, T. G., Dinh, T. N., Tu, J. P., and Dinh, A. T., The boiling crisis phenomenon, Part II: Dryout dynamics and burnout, Exp. Thermal Fluid Sci., vol. 26, pp. 793-810, 2002.

Wayner, P. C., Kao, Y. K., and LaCroix, L. V., The interline heat-transfer coefficient of an evaporating wetting film, Int. J. Heat Mass Transfer, vol. 19, pp. 487-492, 1976.

Yagov, V. V., Is a crisis in pool boiling actually a hydrodynamic phenomenon?, Int. J. Heat Mass Transfer, vol. 73, pp. 265-273, 2014.

Yiantsios, S. G. and Davis, R. H., Close approach and deformation of two viscous drops due to gravity and van der Waals forces, J. Colloid Interface Sci., vol. 144, no. 2, pp. 412-433, 1991. 


\section{APPENDIX: EXTENSION OF LUBRICATION THEORY OF EVAPORATING MENISCUS TO HIGH INTERFACE SLOPES}

This extension has been derived by Mathieu (2003) and follows the original idea of Boender et al. (1991) applied to the lubrication theory of the isothermal CL dynamics. The expression of Boender et al. was rederived recently (Snoeijer, 2006). The approach is based on the analytical solution of the 2D Stokes flow problem in the straight wedge with the opening angle $\phi$. The fluid sink $U$ is placed at its apex (CL position). The boundary conditions mimic the Stokes free-interface problem statement far from the CL, where the slope is large but the evaporation is weak. The flow is directed to the CL where the liquid is evaporated. The boundary conditions reflect (i) the impermeability of the solid substrate $y=0$, no slip at the substrate, (ii) the impermeability of the liquid-gas interface and zero tangential stress at it. The solution to such a problem in terms of the stream function

$$
\Psi(r, \varphi)=-U \frac{\sin (2 \phi-2 \varphi)+2 \varphi \cos (2 \phi)}{2 \phi \cos (2 \phi)-\sin (2 \phi)},
$$

where $(r, \varphi)$ are the polar coordinates with the center in the wedge apex, has been found by Mathieu (2003). The corresponding solution for the liquid pressure at the interface reads

$$
\frac{d p_{L}}{d r}=U \frac{\mu}{r^{3}} \frac{4}{\phi \cos (2 \phi)-\cos \phi \sin \phi} .
$$

Consider now the curved interface with a small curvature. The main assumption (Boender et al., 1991) is that the liquid flow locally is equivalent to that of the straight wedge with the opening angle corresponding to the local slope of the interface (see Fig. 2). The local pressure variation $d p_{L} / d s$ at the liquid-gas interface is thus given by Eq. (A2) where $\phi$ is now a function of $s$. The identity $r=\delta / \phi$ leads to the expression

$$
\frac{d p_{L}}{d s}=-U \mu \frac{3}{\delta^{3}} G
$$

where

$$
G=-\frac{\phi^{3}}{3} \frac{4}{\phi \cos 2 \phi-\cos \phi \sin \phi}
$$

is a correction factor to the conventional lubrication approximation (Oron et al., 1997), where $G(\phi \rightarrow 0)=1$.

By following Delon et al. (2008), we add the slip length and Marangoni effects to Eq. (A3) in Eq. (9) without modifying $G$ as is justified by the fact that these effects are important only in the CL close vicinity where $\phi$ is small and the correction to the lubrication theory is not needed. 